\title{
Multimodality imaging: Bird's eye view from The European Society of Cardiology Congress 2015 London, August 29-September 2, 2015
}

\author{
Jeroen J. Bax, MD, PhD, ${ }^{\text {a }}$ Victoria Delgado, $M D, P h D,{ }^{\text {a }}$ Stephan Achenbach, MD, \\ $\mathrm{PhD},{ }^{\mathrm{b}}$ Udo Sechtem, MD, PhD, ${ }^{\mathrm{c}}$ and Juhani Knuuti, $\mathrm{MD}, \mathrm{PhD}^{\mathrm{d}}$ \\ a Department of Cardiology, Heart Lung Centrum, Leiden University Medical Center, Leiden, \\ The Netherlands \\ ${ }^{b}$ Department of Cardiology, University Hospital Erlangen, Erlangen, Germany \\ c Department of Cardiology, Robert-Bosch-Krankenhaus Stuttgart, Stuttgart, Germany \\ d Turku PET Centre, Turku University Hospital, University of Turku, Turku, Finland
}

doi: $10.1007 / \mathrm{s} 12350-015-0322-1$

This bird's eye view from the 2015 European Society of Cardiology (ESC) congress hosted in London from August 29 to September 2 includes highlighted abstracts on multimodality imaging. At this ESC meeting, 407 imaging abstracts (40\% of abstracts submitted under the topic of imaging) were presented. Four experts on echocardiography (VD), cardiac computed tomography (CT) (SA), cardiac magnetic resonance (CMR) (US), and nuclear imaging (JK) summarized 4 to 7 abstracts in these areas that were of most interest to them. These abstracts were integrated by one of the Editors of the Journal (JB).

\section{ECHOCARDIOGRAPHY}

Speckle tracking echocardiography (STE) continues providing novel insights into cardiac mechanics and prognosis. The Berliner Frauen Risiko evaluation study assessed the association between left atrial (LA) reservoir, conduit and booster pump functions measured with STE and left ventricular (LV) diastolic dysfunction grade in 449 women. ${ }^{1}$ Strain-derived reservoir and conduit functions reduced in parallel to worsening diastolic dysfunction, whereas LA booster pump function increased in grade 1 diastolic dysfunction, and subsequently decreased in grade 2 and 3 diastolic

Reprint requests: Jeroen J. Bax, MD, PhD, Department of Cardiology, Heart Lung Centrum, Leiden University Medical Center, Albinusdreef 2, 2300 RC, Leiden, The Netherlands; j.j.bax@lumc.nl

J Nucl Cardiol 2015;22:1171-8.

$1071-3581 / \$ 34.00$

Copyright () 2015 The Author(s). This article is published with open access at Springerlink.com dysfunction. Compared with LA volume index, strainderived reservoir and conduit functions showed higher sensitivity (73.7\% and $87.2 \%$, respectively) and specificity ( $80.4 \%$ and $75.8 \%$, respectively) to predict LV diastolic dysfunction. These results suggest that changes in LA functions assessed with STE may precede LA remodeling in the early phases of LV diastolic function.

Early diagnosis of arrhythmogenic right ventricular dysplasia (ARVD) using STE was the objective of the study presented by Teske and coworkers. ${ }^{2}$ The electromechanical interval, defined as the time difference between first ECG-detected deflection of the QRS complex and the onset of shortening of the basal, mid, and apical segments of the right ventricular (RV) free wall, was measured as a surrogate of activation delay in 44 mutation-positive ARVD patients, 31 mutation carriers without phenotype of ARVD, and 30 healthy controls. The electro-mechanical interval was associated with the occurrence of ventricular tachyarrhythmias. From the healthy controls, a cut-off value $>100 \mathrm{~ms}$ was derived to define an abnormal electro-mechanical interval. Mutation-positive ARVD patients had an abnormal electro-mechanical interval in all RV segments, whereas only $55 \%$ of mutation carriers without ARVD phenotype showed an abnormal electro-mechanical interval in the basal RV segment. After a median follow-up of $3.8 \pm 2.8$ years, patients of the mutation-positive and the mutation carriers without phenotype of ARVD groups with an abnormal electro-mechanical interval at the basal segment of the RV free wall showed a significantly higher burden of ventricular arrhythmias.

The results of a sub-study from the EchoCRT trial (Cardiac resynchronization therapy in heart failure with narrow QRS complex) were presented, focusing on the 
prognostic implications of persistent or worsened LV dyssynchrony after CRT implantation. ${ }^{3}$ Of 614 patients with echocardiographic follow-up at 6 months after CRT implantation, 77\% showed persistent or worsened LV dyssynchrony $(\geq 130 \mathrm{~ms}$ as measured with STE or $\geq 80 \mathrm{~ms}$ using tissue Doppler imaging). Patients with persistent or worsened LV dyssynchrony showed a significantly increased risk of all-cause mortality and heart failure hospitalization (hazard ratio 1.54, 95\% confidence interval 1.03-2.30; $P=.02$ ) compared with patients with reduced LV dyssynchrony, confirming observational results in heart failure patients with wide QRS complex. ${ }^{4}$

Three-dimensional transthoracic echocardiography (3D TTE) attracts significant attention. Tricuspid valve regurgitation in patients with $\mathrm{RV}$ pacing leads has poor prognosis. ${ }^{5}$ In 145 patients with pacemaker or implantable cardioverter defibrillator (ICD), the mobility of the RV pacing lead and its position relative to the tricuspid valve annulus was assessed with 3D TTE. At follow-up after device implantation, 30\% presented with grade 3 or more tricuspid regurgitation. Patients were classified as having low risk for significant tricuspid regurgitation if the lead was freely mobile without interfering with the leaflet movement and positioned in the center of the tricuspid valve annulus or in the commissure of the tricuspid leaflets. In contrast, patients with high risk of developing significant tricuspid regurgitation had more frequently fixed leads interfering with leaflet mobility and more frequently showed leaflet perforation. Three-dimensional analysis of the tricuspid valve and the underlying pathophysiology of tricuspid regurgitation may help to personalize treatment of patients with symptomatic severe tricuspid regurgitation. The use of dedicated software to create a 3D model that can subsequently be printed may help to decide on the repair approach. Muraru and coworkers presented the first 3D printed model of the tricuspid valve. ${ }^{6}$ Coordinates of the tricuspid annulus and leaflets were imported into Mesh-Lab (Visual Computing Lab ISTI-CNR) software to build a model which was converted to stereolithographic file format and printed in $3 \mathrm{D}$ in 30 minutes (Figure 1). The 3D printed tricuspid valve permitted better perception of the complex 3D geometry.

\section{COMPUTED TOMOGRAPHY}

In cardiac $\mathrm{CT}$, most of the presented research at the ESC Congress 2015 was in relation to clinical applications, and relatively few abstracts concerned new technical developments. The use of "virtual" fractional flow reserve, derived from the anatomic information provided by coronary CT angiography (CTA), received most attention. The PLATFORM study ${ }^{7}$ was designed to evaluate the utility of coronary CTA with fractional flow reserve (FFR-CT) to serve as a "gatekeeper" for invasive angiography. A total of 584 patients with new onset chest pain were recruited in 11 centers in Europe, with core laboratory evaluation in the United States. The patients were divided in 2 cohorts: a "non-invasive workup first" cohort of 204 individuals, and a "planned invasive angiography" cohort of 380 patients, depending on the management planned by the treating physician. In both cohorts, patients were randomized to either the originally planned test, or to coronary CTA with FFR-CT (in the presence of 30\% or more luminal stenosis). The primary outcome measure was the rate of invasive coronary angiograms that did not show obstructive disease in the cohort that had been planned to undergo invasive angiography. Indeed, the use of coronary CTA (plus FFR-CT in 117 cases) resulted in a rate of invasive coronary angiograms showing no stenosis of $12.4 \%$ (24/193), as compared to $73.3 \%$ (137/ 187 ) in those who underwent the initially planned invasive angiogram directly, without prior CT. In the cohort of 204 patients for whom a non-invasive workup

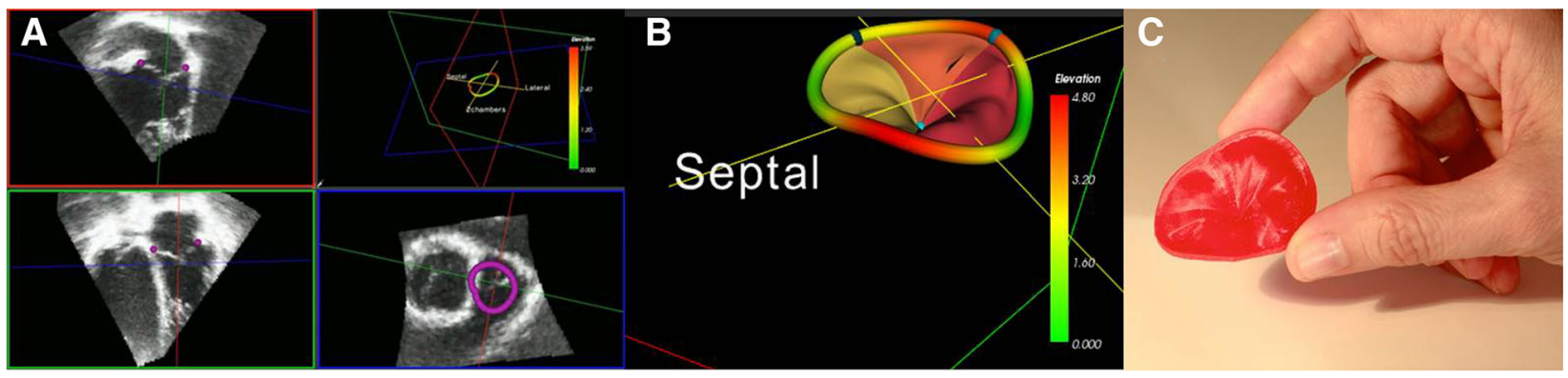

Figure 1. Three-dimensional printing of the tricuspid valve. From three-dimensional transthoracic echocardiographic data of the tricuspid valve, coordinates of the leaflets and annulus are demarcated (A) and imported to dedicated software to create the model (B) which is converted to stereolithographic file format and printed in 3D $(\mathbf{C})$. Reproduced with permission from Muraru et $\mathrm{al}^{6}$. 
was initially planned, there was no significant difference in the rate of invasive angiograms showing no stenoses (6.0\% without CTA/FFR-CT vs. $12.5 \%$ with CTA/FFR$\mathrm{CT}, P=.95)$. However, this was not a primary outcome measure. Average radiation exposure was not increased by the use of CTA/FFR-CT in either cohort. Ninety-day major adverse cardiac event (MACE) rates were not increased in those patients who underwent CTA rather than invasive angiography. All in all, the study reassuringly showed that CTA/FFR-CT can reduce the number of unnecessary invasive angiograms, and specifically reduce the number of angiograms showing no stenosis, in patients with suspected coronary disease-hence well serving its "'gatekeeper'" role.

Numerous abstracts dealt with the prognostic value of coronary CTA. Bom et al evaluated 1551 patients with low-to-intermediate risk of coronary artery disease who underwent coronary CTA as well as calcium scoring. ${ }^{8}$ During a median follow-up of 637 days, 23 patients $(1.5 \%)$ experienced an event: late revascularizations (16/23), myocardial infarction (4/23), and death (3/23). Both coronary calcium scoring and coronary CTA had prognostic value. The multivariable analysis, adjusting for risk factors and coronary calcium score, demonstrated an independent prognostic value of coronary CTA (Figure 2).

Marwan et al reported the results of the German cardiac CT registry regarding the presence of coronary artery lesions in coronary CTA of 2016 individuals without coronary calcium. ${ }^{9}$ In $18 \%$ of these individuals, non-obstructive coronary lesions and in $2.3 \%$, obstructive lesions were found, emphasizing that coronary atherosclerosis can occur even in patients without coronary calcium.

An increasingly important area is the use of cardiac CT for non-coronary interventions, for example, in heart valve disease. Fonseca et al presented a series of 131 patients who underwent transcatheter aortic valve implantation with a balloon-expandable $(36 \%)$ or selfexpanding $(64 \%)$ prosthesis. $^{10} 53 \%$ of patients had paravalvular regurgitation after the procedure, which was mild in $46 \%$ and moderate in $8 \%$. Aortic valve calcium was predictive of post-implant regurgitation, with an optimal cut-off value of $157 \mathrm{~mm}^{3}$ of calcium measured with a lower threshold of $850 \mathrm{HU}$ to accept a structure as "calcium." The degree of aortic valve calcification (optimum cut-off: $267 \mathrm{~mm}^{3}$ ) was also a predictor for the need to perform balloon post-dilatation. Mitral valve interventions may also profit from CT imaging. In 25 patients planned for edge-to-edge mitral valve repair, CTA and transesophageal echocardiography showed excellent agreement regarding parameters such as leaflet length, annulus diameters, and left ventricular dimensions. ${ }^{11}$ Finally, CT imaging is important in patients considered for transcatheter LA appendage occlusion, and CT may be the preferred method for assessment of mean diameter of the LA appendage orifice. ${ }^{12}$
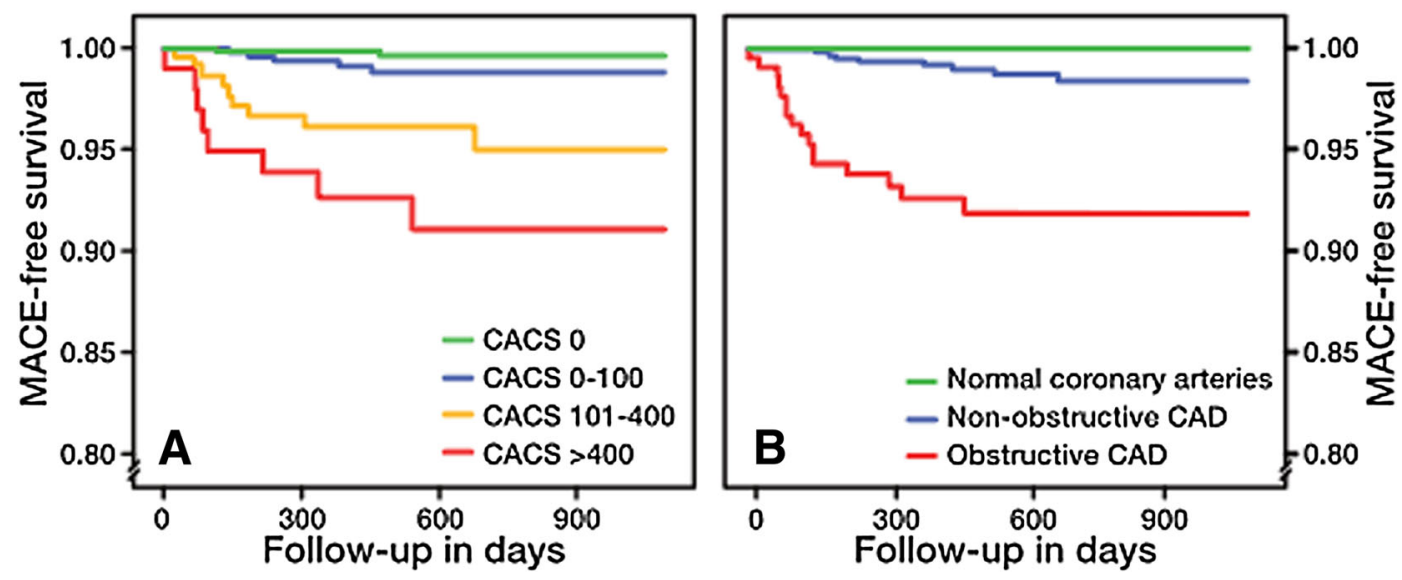

Figure 2. Major adverse cardiac event (MACE)-free survival in a cohort of 1551 patients with chest pain and a low-to-intermediate pre-test probability for coronary artery disease followed for a median of 637 days. A total of 23 MACE occurred, in the majority late revascularizations $(n=16)$. Left prognostic value of the calcium score, right prognostic value of coronary computed tomography angiography (CTA). In multivariable analysis, obstructive plaque in coronary CTA added significantly to the calcium score and risk factors. $C A C S$, coronary artery calcium score; $C C T A$, coronary computed tomography angiography. Reproduced with permission from Bom et $\mathrm{al}^{8}$. 


\section{CARDIAC MAGNETIC RESONANCE IMAGING}

Important topics in research with cardiac magnetic resonance (CMR) at the ESC Congress 2015 included assessment of diffuse myocardial fibrosis, advanced diagnosis of coronary artery disease, and risk stratification for ICD implantation.

Validation of pre-contrast T1 mapping values and myocardial extracellular volume measurement with CMR techniques as surrogates of interstitial diffuse fibrosis was the objective of several studies including patients with different cardiomyopathies. ${ }^{13-15}$ In patients with dilated cardiomyopathy, longer pre-contrast T1 mapping values were significantly correlated with larger amounts of extracellular volume and larger histological collagen volume fraction, indicating more extensive LV fibrosis..$^{14,15}$ In addition, in 531 consecutive patients referred for CMR for other reasons than hypertrophic cardiomyopathy, Kammerlander et al showed that increasing myocardial extracellular volume was independently associated with increased risk of heart failure hospitalization and cardiovascular death. ${ }^{13}$ In patients with acute ST-segment elevation myocardial infarction, magnitude and changes in myocardial extracellular volume (assessed with T1 mapping) in areas remote from the infarct core, were associated with changes in LV ejection fraction (EF). ${ }^{16}$ In 171 ST-segment elevation myocardial infarction patients, extracellular volume of the remote area at baseline (2 days after index infarction) and at 6 months follow-up did not change significantly (from $25.6 \pm 3.1 \%$ to $25.5 \pm 2.8 \%$ ), whereas LVEF increased from $55.6 \pm 9.3 \%$ to $62.7 \pm$ $9.4 \%$. A change in extracellular volume was inversely associated with a change in LVEF $(r=-0.23 ; P=$ .011). Moreover, an increase in extracellular volume of the remote area of $\geq 1 \%$ at 6 months follow-up was observed in $43 \%$ of patients. ${ }^{17}$ Compared with patients in whom extracellular volume did not change, patients with increased extracellular volume showed "higher risk" characteristics: older age, higher peak troponin, more frequent microvascular obstruction and reduced LVEF, and larger LV volumes at follow-up. This work demonstrates that the adverse process of LV remodeling post-ST-segment elevation myocardial infarction can be detected and quantified non-invasively with CMR.

Coronary microvascular disease is characterized by angina, positive tests for ischemia, and the absence of epicardial coronary stenoses. CMR can be useful in these patients to determine coronary flow reserve by measuring coronary sinus flow at rest and during pharmacologic stress, and then myocardial perfusion reserve is calculated as the ratio between stress coronary sinus flow/baseline coronary sinus flow in 245 consecutive patients. Patients with a myocardial perfusion reserve below or equal to the mean (2.75) had worse outcome compared to patients with preserved myocardial perfusion reserve $(>2.75) .{ }^{18}$ Furthermore, patients with microvascular dysfunction often experience resting chest pain due to concomitant microvascular spasm. Coronary spasm including microvascular spasm can be provoked by intracoronary acetylcholine. This test revealed microvascular spasm in 59 out of 125 patients, whereas epicardial spasm was elicited in 28 of 125 patients. ${ }^{19}$ Stress CMR showed a reversible adenosine-induced subendocardial perfusion defect in 58 out of these 125 patients (Figure 3).

Patients with dilated cardiomyopathy and $\mathrm{LVEF}<35 \%$ are candidates for ICD implantation as primary prevention of sudden cardiac death. The incremental prognostic value of LVEF and the presence of myocardial scar assessed with CMR were evaluated in 270 patients with dilated cardiomyopathy. ${ }^{20}$ During 2.5 years of follow-up, 68 presented with ventricular tachyarrhythmias and sudden cardiac death. Patients with events had lower LVEF $(29 \pm 10$ vs. $32 \pm 9 \%$, $P=.003)$ and higher frequency of myocardial scar (67 vs. $44 \%, P=.0009)$ than patients who did not present with events.

In addition, an interesting multicenter trial studied whether the extent and heterogeneity of late gadolinium enhancement (LGE) areas (identifying the infarct border zone) predicted appropriate ICD therapy in 55 patients with ischemic cardiomyopathy and 62 with dilated cardiomyopathy. ${ }^{21}$ During a mean follow-up of 4 years, patients with larger (above the median) compared to smaller border zones had higher rates of ICD therapy, whereas total LGE burden was similar between groups with and without ICD therapy. The importance of the size of the border zone surrounding the scar was confirmed in another study of 138 ST-segment elevation myocardial infarction patients who were studied by CMR within 1 week post-infarction. ${ }^{22}$ In a multivariate model that included clinical and other established prognostic parameters, the extent of the border zone was an independent predictor of the combined clinical endpoint (all-cause death, re-infarction, new congestive heart failure within 1 year of infarction). Thus, border zone size may be a marker for risk stratifying patients eligible for primary prevention ICD therapy.

\section{NUCLEAR CARDIOLOGY}

In nuclear cardiology, not only a large fraction of abstracts focused on coronary artery disease and myocardial perfusion imaging (MPI) but also other methods such as innervation imaging gained increasing attention. 

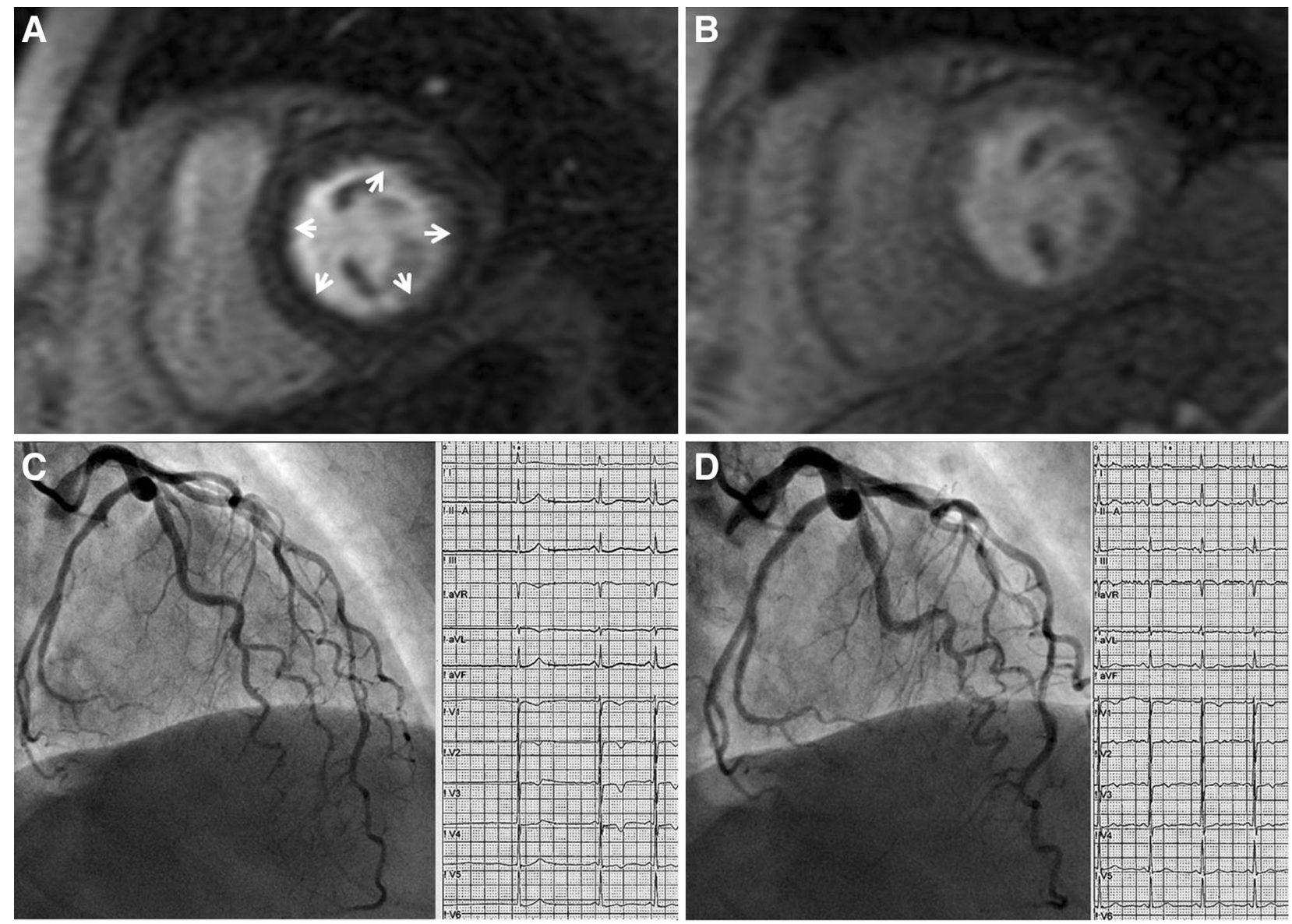

Figure 3. Stress CMR to assess microvascular disease. Example of a 72-year-old woman with angina complaints during rest and negative troponin. After reaching a peak exercise of $75 \mathrm{~W}$ during electrocardiogram stress testing, the patient developed chest pain. There was a ST-segment depression in I, II, aVF, V4 to V6 leads. CMR showed circular subendocardial hypoenhancement during adenosine stress (A, arrows). Perfusion at rest was normal (B). Acetylcholine testing at $100 \mu \mathrm{g}$ showed diffuse mild constriction of the epicardial left coronary artery $(\mathbf{C})$ which was accompanied by ST-segment depression in V1 to V3 leads. Following nitroglycerin injection into the left coronary artery, the epicardial coronary artery appeared wider (D) and ST-segment abnormalities resolved. Reproduced with permission from Ong et $\mathrm{al}^{19}$.

Al-Mallah et $\mathrm{al}^{23}$ investigated the incremental prognostic significance of coronary flow reserve in 2645 consecutive patients with known or suspected coronary artery disease using positron emission tomography (PET, stress, and rest) and rubidium-82. During a median follow-up of 1.4 years, data on cardiac death or myocardial infarction were collected. The lowest tertile of coronary flow reserve $(<1.8)$ was associated with a 3.7-fold increase in the risk of events, as compared with the highest tertile. This remained significant after adjusting for clinical variables, perfusion defect size, and resting LVEF. The study further emphasizes the clinical value of absolute quantification of myocardial perfusion and perfusion reserve over standard visual assessment.
The prognostic value of single-photon emission computed tomography (SPECT) MPI was addressed in 564 patients who were evaluated for renal transplantation. ${ }^{24}$ During a median follow-up of 43.5 months, 122 patients died (9.6\% of cardiovascular causes). Compared with patients without ischemia, patients with mild (5-10\% of the LV myocardium) and with substantial ischemia $(>10 \%)$ showed a significantly increased mortality risk (respective hazard ratios: 2.27 and 2.44). However, patients with substantial ischemia had lower risk for cardiovascular death than patients with mild ischemia, possibly because of more frequent revascularizations.

The detection of vulnerable plaques using noninvasive imaging has gained a lot of interest over the 

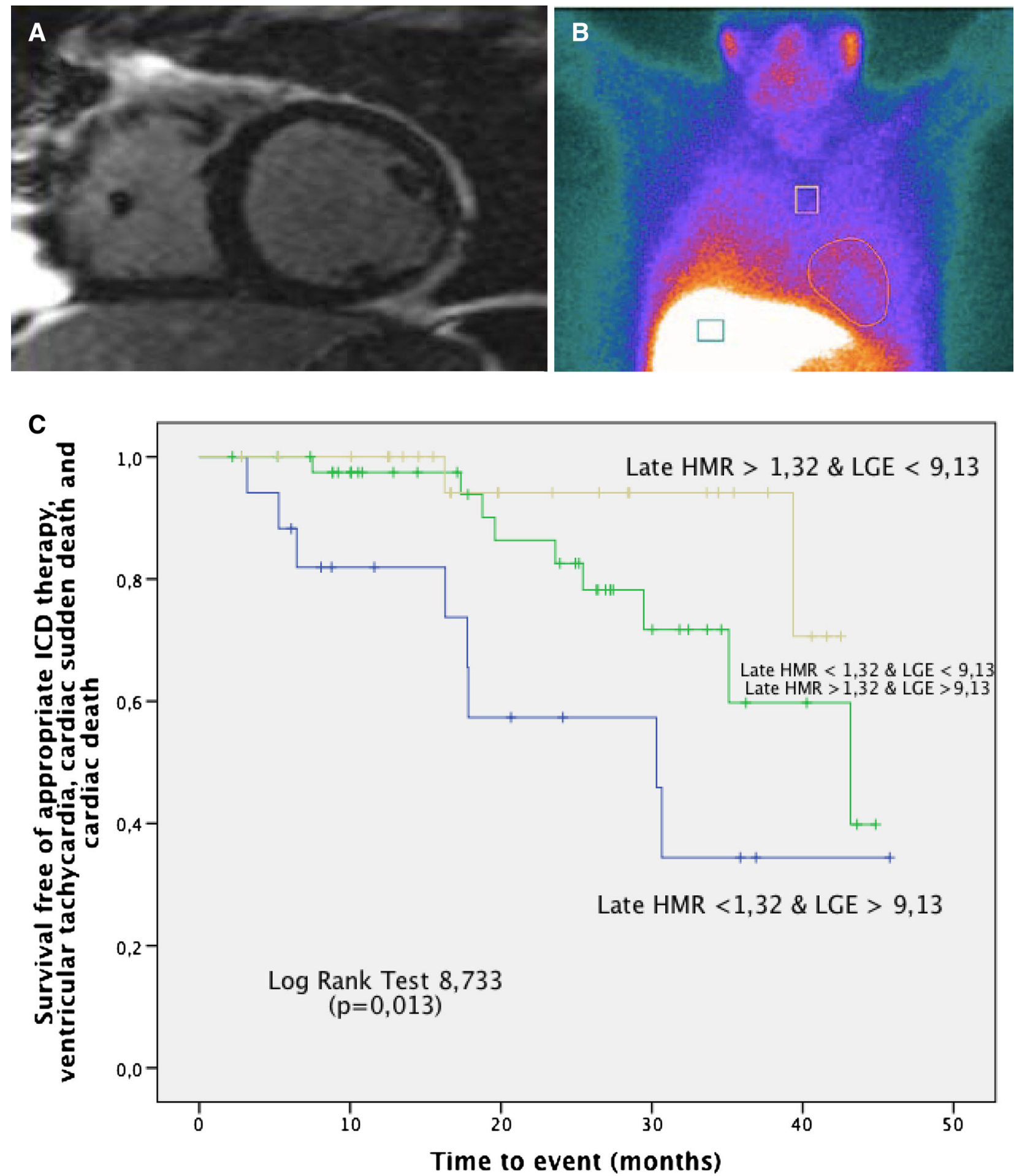

Figure 4. Combination of fibrosis imaging by cardiac magnetic resonance (CMR) (A) and late and I-123-iodine metaiodobenzylguanidine (MIBG) uptake (B) and outcomes (C): patients with larger amount of late gadolinium enhancement (LGE) on CMR and lower MIBG uptake on SPECT have worse prognosis than the other groups of patients. Reproduced with permission from García-González et $\mathrm{al}^{26}$. 
recent years. PET imaging using F-18-fluorodeoxyglucose (FDG) has been used in numerous studies to detect vascular inflammation in the aorta and carotid arteries. Recently, studies have used FDG and F-18-fluoride to detect active plaques in human coronary arteries. Locorotondo et $\mathrm{al}^{25}$ compared the characteristics of unstable coronary artery plaques detected by intracoronary optical coherence tomography and FDG PET imaging in 20 consecutive patients with single-vessel coronary artery disease. Coronary FDG uptake was enhanced in patients with non-ST-elevation acute coronary syndrome as compared to patients with stable coronary artery disease, with a trend toward higher activity in patients with inflamed thin cap fibroatheromas. The majority of patients (88\%) without inflamed thin cap fibro-atheromas and low FDG uptake had stable coronary artery disease, while all patients with inflamed thin cap fibro-atheromas and high FDG uptake had non-ST-elevation acute coronary syndrome suggesting that FDG uptake in plaques is associated with inflammation and vulnerability.

Several studies focused on cardiac innervation imaging using SPECT and I-123-iodine metaiodobenzylguanidine (MIBG) or PET with [11C]hydroxyephedrine (HED). Garcia Gonzalez et $\mathrm{al}^{26}$ performed scar imaging with CMR and planar MIBG imaging in 86 heart failure patients who were referred for ICD implantation. The endpoints included appropriate ICD shocks, ventricular tachycardia, and cardiac death; 19 patients $(22 \%)$ reached the endpoint. These patients had larger scar areas on late gadolinium enhancement (LGE $\geq 9.13 \%$ ) and reduced MIBG uptake (heart to mediastinum ratio $\leq 1.32$ ). The combination of LGE CMR and MIBG imaging improved the risk stratification (Figure 4).

Exercise training has beneficial effects in heart failure patients. Valborgland et al evaluated whether cardiac MIBG uptake would improve after 3 months of exercise training. ${ }^{27}$ The study enrolled 23 patients who were randomized to regular exercise, continuous training, and interval training. All patients underwent MIBG imaging before and after the training period. The authors reported no differences between the groups, suggesting that alterations in sympathetic innervation are not related to the beneficial effects of exercise training in heart failure patients.

Finally, the effect of beta-blocker therapy on cardiac sympathetic nerve function in patients with idiopathic pulmonary arterial hypertension was studied by Rijnierse et al. ${ }^{28}$ Patients with pulmonary arterial hypertension and right ventricular failure are known to suffer from impaired cardiac sympathetic innervation, which may be related to the severity of the disease. The authors investigated 18 patients with pulmonary hypertension using [11C]HED PET in a prospective, double- blind placebo-controlled crossover trial. A heterogeneous pattern of sympathetic nerve dysfunction was noted, particularly in the right ventricle and septum. Beta-blocker therapy resulted in increased septum and right ventricle [11C]HED uptake, suggesting a positive effect on regional neuronal remodeling.

\section{Disclosure}

The Department of Cardiology of the Leiden University Medical Center received research grants from Biotronik, Edwards Lifesciences, Medtronic and Boston Scientific. The remaining authors have nothing to disclose.

\section{Open Access}

This article is distributed under the terms of the Creative Commons Attribution 4.0 International License (http:// creativecommons.org/licenses/by/4.0/), which permits unrestricted use, distribution, and reproduction in any medium, provided you give appropriate credit to the original author(s) and the source, provide a link to the Creative Commons license, and indicate if changes were made.

\section{References}

1. Brecht A, Oertelt-Prigione S, Regitz-Zagrosek V, Baumann G, Knebel F, Stangl V. Alterations of phasic left atrial strain in patients with pre-clinical diastolic dysfunction: 2D speckle tracking echocardiography-derived results from the BEFRI-echo study. Eur Heart J 2015;36:945.

2. Teske AJ, Mast TP, van der Heijden JF, Loh P, Cramer MJ, Doevendans PA, et al. Predictive value of local prolonged electromechanical interval by echocardiography in the concealed stage of arrhythmogenic right ventricular dysplasia/cardiomyopathy. Eur Heart J 2015;36:1022.

3. Gorcsan J III, Sogaard P, Bax JJ, Singh JP, Abraham WT, Borer JS, et al. Association of persistent or worsened echocardiographic dyssynchrony with unfavourable clinical outcomes in heart failure patients with narrow QRS width: A subgroup analysis of the EchoCRT trial. Eur Heart J 2015. doi:10.1093/eurheartj/ehv418.

4. Auger D, Bleeker GB, Bertini M, Ewe SH, van Bommel RJ, Witkowski TG, et al. Effect of cardiac resynchronization therapy in patients without left intraventricular dyssynchrony. Eur Heart J 2012;33:913-20.

5. Hamdanchi A, Asadi Y, Otto S, Hoyme M, Jung C, Lauten A, et al. A cross-sectional study of endocardial lead-related tricuspid regurgitation: Towards proposing a new practical 2D/3D echocardiographic approach for better risk stratification. Eur Heart J 2015;36:595.

6. Muraru D, Veronesi F, Dequal D, Maddalozzo A, Jenei C, Addetia $\mathrm{K}$, et al. Three-dimensional printing of tricuspid valve using transthoracic echocardiography. Eur Heart J 2015;36:429.

7. Douglas PS, Pontone G, Hlatky MA, Patel MR, Norgaard BL, Byrne RA, et al. Clinical outcomes of fractional flow reserve by computed tomographic angiography-guided diagnostic strategies vs. usual care in patients with suspected coronary artery disease: The prospective longitudinal trial of FFRct: outcome and resource impacts study. Eur Heart J 2015. doi:10.1093/eurheartj/ehv444. 
8. Bom MJ, van der Zee PM, van der Zant FM, Knol RJJ, Cornel JH. Independent prognostic value of coronary artery calcium score and coronary computed tomography angiography in an outpatient cohort of low to intermediate risk chest patients. Eur Heart J 2015;36:891.

9. Marwan M, Achenbach S, Schneider S, Schumermund A, Korosoglou G, Hausleiter J, et al. Coronary CT angiography in patients with a zero agatston score: Results from the German cardiac CT registry. Eur Heart J 2015;36:765.

10. Fonseca P, Almeida C, Almeida C, Bettencourt N, Sampaio F, Ferreira $\mathrm{N}$, et al. Aortic valve calcium volume predicts paravalvular regurgitation and the need for balloon post-dilatation after transcatheter aortic valve implantation. Eur Heart J 2015;36:866.

11. Renker M, Rixe J, Schneck F, Moellmann S, Walther C, Rolf A, et al. Computed tomography angiography compared to transesophageal echocardiography for assessing mitral valve parameters prior to percutaneous edge-to-edge mitral valve repair. Eur Heart J 2015;36:771

12. Iriart $\mathrm{X}$, Selmi W, Jalal Z, Thambo JB. Left atrial appendage sizing for percutaneous occlusion with amplatzer cardiac plug: A multumodality imaging approach. Eur Heart J 2015;36:772.

13. Kammerlander A, Pfaffenberger S, Zotter-Tufaro C, Bachmann A, Aschauer S, Duca F, et al. T1 mapping by cardiac magnetic resonance imaging: From histological validation to clinical implication. Eur Heart J 2015;36:354.

14. Nakamori S, Dohi K, Ishida M, Goto Y, Omori T, Fujimoto N, et al. Pre-contrast T1-mapping and extracellular volume mapping for the assessment of myocardial fibrosis: A validation with histologic sample. Eur Heart J 2015;36:248.

15. Tateishi E, Noguchi T, Morita Y, Ogawa H, Yasuda S. Clinical impact and usefulness of native $\mathrm{T} 1$ mapping in patients with idiopathic dilated cardiomyopathy. Eur Heart J 2015;36:246.

16. Carberry J, Carrick D, Haig C, Rauhalammi SM, Ahmed N, McEntegart M, et al. Changes in remote extracellular volume and left ventricular ejection fraction during longitudinal follow-up are inversely associated in survivors of acute STEMI. Eur Heart J 2015;36:84.

17. Carberry J, Carrick D, Haig C, Rauhalammi SM, Ahmed N, McEntegart M, et al. Natural history and clinical significance of infarct zone extracellular volume and remodelling in survivors of acute STEMI. Eur Heart J 2015;36:244.

18. Jariwala N, Bauml M, Romano S, Rangarajan V, Chacko S, Chung $\mathrm{J}$, et al. Prognostic significance of global myocardial perfusion reserve measured using coronary sinus flow during stress cardiovascular magnetic resonance imaging. Eur Heart J 2015;36:528.

19. Ong PE, Pirozzolo G, Bentz K, Athanasiadis A, Hill S, Schaeufele $\mathrm{T}$, et al. Subendocardial stress perfusion defects on cardiovascular magnetic resonance in patients with angina and unobstructed coronaries are frequently related to functional coronary vasomotor abnormalities. Eur Heart J 2015;36:529.

20. Pontone G, Andreini D, Solbiati A, Guglielmo M, Mushtaq S, Baggiano A, et al. Comparison of transthoracic echocardiography versus cardiac magnetic for implantable cardioverter defibrillator therapy in primary prevention strategy dilated cardiomyopathy patients. Eur Heart J 2015;36:684.

21. Jablonowski R, Chaudhry U, van der Pals J, Engblom H, Arheden $\mathrm{H}$, Heiberg E, et al. Cardiac magnetic resonance can predict appropriate primary prevention ICD-therapy in ischemic and dilated cardiomyopathy patients using late gadolinium enhancement heterogeneity. Eur Heart J 2015;36:685.

22. Eitel C, Bode F, Desch S, Thiele H, Eitel I. Prognostic value of peri-infarct tissue heterogeneity in reperfused STEMI. Eur Heart J 2015;36:685

23. Al-Mallah M, Ahmed A, Aljizeeri A, Suleiman I. Incremental prognostic value of noninvasive coronary flow reserve. Eur Heart J 2015;36:857.

24. Helve S, Laine M, Helantera I, Sinisalo J, Lammintausta O, Lehtonen $\mathrm{J}$, et al. Myocardial perfusion imaging predicts mortality in patients evaluated for kidney transplantation. Eur Heart $\mathbf{J}$ 2015;36:857.

25. Locorotondo G, Danza ML, Burzotta F, Porto I, Niccoli G, Leone $\mathrm{AM}$, et al. Identification of unstable coronary artery plaques by intracoronary optical coherence tomography and positron emission tomography. Eur Heart J 2015;36:81.

26. Garcia-Gonzalez P, Cozar-Santiago P, Fabregat-Andres O, Estornell-Erill J, Sanchez-Jurado R, Bochard-Villanueva B, et al. Combination of fibrosis by cardiac magnetic resonance and late heart-to-mediastinum ratio by cardiac 123-I MIBG imaging improves risk stratification in candidates for primary prevention implantable cardioverter defibrillator. Eur Heart J 2015;36:13.

27. Valborgland T, Isaken K, Munk PS, Larsen AI. Heart neuronal function assessed with I-123 MIBG after 3 months of exercise training in heart failure patients. Eur Heart J 2015;36:80.

28. Rijnierse MT, van Campen JS, De Boer K, Harms HJ, De Man FS, Lammertsma AA, et al. Effect of beta-blocking therapy on cardiac sympathetic nerve function in patients with idiopathic pulmonary arterial hypertension. Eur Heart J 2015;36:12 